\title{
Analysis of Ocean Reverberation Characteristics Based on the Vector Hydrophones

\author{
Bing Jia ${ }^{1, a}$, Yunfei Chen ${ }^{1, b}$, Yang Zhang ${ }^{1}$, Feng Gao ${ }^{1}$ \\ ${ }^{1}$ Science and Technology on Underwater Test and Control Laboratory, Dalian, 116013, China \\ aemail:jiabing.robin@gmail.com, bemail: yunfeidlut@163.com
}

Keywords: Vector Hydrophone; Ocean Reverberation; Measurement

\begin{abstract}
Focusing on the disturbance of ocean reverberation in active sonar detection, this article researched the characteristics of vector ocean reverberation when vector hydrophones were the receivers. By experiments and theoretic analyses, scalar and vector ocean reverberation was gained when transmitting signal with different frequencies, impulse widths, receivers at different depths, and the source levels and laws of energy attenuation were analyzed statistically. By comparison, reverberation received by vector hydrophones was found to have characteristic of lower reverberation levels and faster attenuation, thus, vector hydrophones have the ability of anti-reverberation.
\end{abstract}

\section{Introduction}

In active sonar detection, ocean reverberation is the primary background disturbance, which affects the ability of target detection and identification of active sonar greatly. Based on the scalar information of ocean reverberation received by pressure hydrophones, laws of energy evolvement, frequency spectrum, statistical characteristics of phase, correlation of time and space are studied in traditional reverberation research[1][2]. With the abroad application of vector hydrophones, vector parameters of underwater sound field can be measured, such as point velocity, pressure gradient, and acceleration. Methods of signal processing evolve from single scalar method processing to scalar and vector methods combined processing. Measuring and analyzing characteristics of energy and frequency of ocean vector reverberation field, conduces to improving abilities of detection and identification of underwater acoustic devices based on vector hydrophones[3][4].

\section{Equivalent plane wave reverberation level}

Reverberation follows on the heels of impulses transmitted by active sonar system, and is like a series of long, slowly attenuating, trembling sounds. In the active sonar equation, reverberation is denoted by RL----equivalent plane-wave reverberation level[1].

Defining now the equivalent plane-wave reverberation level RL as the intensity, in decibel, of an axially incident plane wave producing the same hydrophone output as the observed reverberation, we obtain

$$
R l=10 \log \frac{I}{I_{0}}
$$

Where $\mathrm{I}$ is the intensity of the plane wave, $I_{0}$ is the reference intensity of a plane wave whose virtual pressure is $1 \mathrm{uPa}$. Therefore $\mathrm{RL}$ describes the strength of reverberation disturbance when reverberation is the primary limitation of active sonar system.

\section{Vector hydrophones}

In the ocean channel, the sound field has information of scalar pressure and vector particle velocity, which approximately meets the acoustic Ohm law, also pressure and particle velocity are at the same phase. Vector hydrophones could gain the pressure and particle velocity information of the underwater sound field by one measuring action. The measured pressure $p(\mathbf{r}, t)$ and the three 
orthogonal subsets of particle velocity $\mathbf{v}(\mathbf{r}, t)$ come as following [5]:

$$
\begin{aligned}
& p(\mathbf{r}, t)=A(\mathbf{r}) \exp \{-i[\omega t-\varphi(\mathbf{r})]\} \\
& \mathbf{v}(\mathbf{r}, t)=\frac{1}{\rho_{0}(\mathbf{r}) \omega}\left[\nabla \varphi(\mathbf{r})-i \frac{\nabla A(\mathbf{r})}{A(\mathbf{r})}\right] p(\mathbf{r}, t) \\
& =v(\mathbf{r}, t) \cdot \mathbf{u}
\end{aligned}
$$

Where $\emptyset \in\left[0, z_{u}\right)$ the horizon azimuth angle of the incident sound wave is, $\theta \in[0, u)$ is the angle between incident sound wave and $\mathrm{Z}$ axis.

In the equation (4), it says that vector hydrophones can gain the pressure and the three orthogonal subsets of particle velocity information of the sound field, whatever the size one hydrophone may take; it has nature directivity of the $\cos \theta$ or $\sin \theta$ form. The advantage of this kind nature directivity enables vector hydrophones to have ability to restrain reverberation.

\section{Methods of signal processing}

Based on the energy attenuation as a function of transmitting signal, measurements and analyses of characteristics of vector reverberation was conducted by comparing measured results of pressure hydrophones and vector hydrophones, where the signal processing comes in three steps: sensitivity compensating, transmitting source level compensating, abstraction of reverberation level and envelops.

(1) Sensitivity compensating

Voltage sensitivity levels of hydrophones in the free field are defined $M_{y_{1} 1}, M_{x_{1}}, M_{y 1} d B$, they follows that

$$
M=20 \lg \left[\frac{e_{o c} / p_{f}}{\left(e_{o c} / p_{f}\right)_{r e f}}\right]
$$

Where $\mathrm{M}$ in $\mathrm{dB}$ is voltage sensitivity in the free field; ${ }_{\infty} / \mathrm{p}_{\mathrm{f}}$ in $\mathrm{V} / \mu \mathrm{Pa}$ is the virtual value of the hydrophone; $\left(\omega_{w} / p_{f}\right)_{\text {ref }}$ in $W / \mu \mathrm{Pa}_{\mathrm{a}}$ is the reference value of the sensitivity.

Thus signals sampled by hydrophones in voltage could be transformed into pressure levels in $\mathrm{dB}$ directly.

(2) Transmitting source compensating

Transmitting source levels affect reverberation levels directly, the higher source levels, the higher reverberation levels. To compare reverberation under different transmitting frequencies, their transmitting source levels must be compensated to the same level.

(3) Abstraction of reverberation levels and envelopes.

Reverberation levels' characteristics of energy and changes with time could be gained by studying the pressure envelopes of reverberation levels. After sensitivity and source level compensation to reverberation wave data, abstract all peak points in each wave cycle, then smooth these peak points, finally gain the envelops. By comparing envelopes of reverberation under different transmitting frequencies and impulse widths, functions could be gained, that characteristics of reverberation energy attenuation is function of transmitting frequencies and impulse widths. 


\section{Results of data processing}

Experiment was conducted in the sea of Dalian, where the water depth is 50 meters, with seabed of mud and sand. To gain the initial receiving signal, intercept the sampled vector reverberation signal by transmitting impulse cycles, then filter and compensate sensitivity. Fig 1 shows the initial waveform of reverberation signal under transmitting frequency $1 \mathrm{kHz}$, impulse width $50 \mathrm{~ms}$.

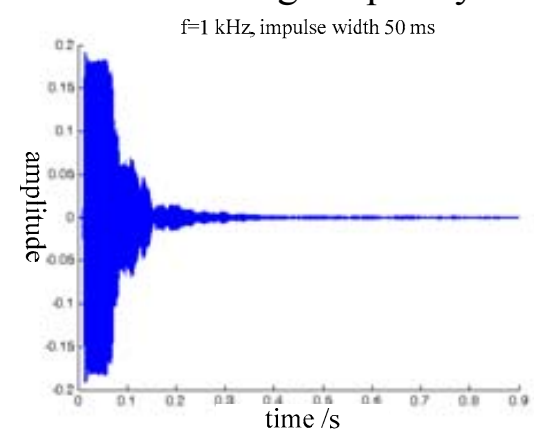

Fig.1. Initial waveform of reverberation signal

In Fig 2, a comparison is made between fluctuations of scalar and vector reverberation under single transmitting frequency $1 \mathrm{kHz}$, impulse width $\mathrm{T}=50 \mathrm{~ms}$. It can be seen that vector reverberation attenuates faster than scalar reverberation at $1 \mathrm{kHz}$, which contributes to the space gain of the vector hydrophones. In Fig 3, a comparison is made between fluctuations of scalar and vector reverberation under broadband modulated frequency $2.5-3.5 \mathrm{kHz}$, impulse width $50 \mathrm{~ms}$. Again vector reverberation attenuates faster than scalar reverberation.

Differences of energy attenuation between scalar reverberation and vector reverberation have just been studied, under both single frequency and broadband modulated frequency transmitting signals. Considering that reverberation changes when frequency and impulse width change, the following part will focus on how the reverberation envelopes change with transmitting frequency and impulse width, comparisons between scalar reverberation and vector reverberation will also be paid attention to.

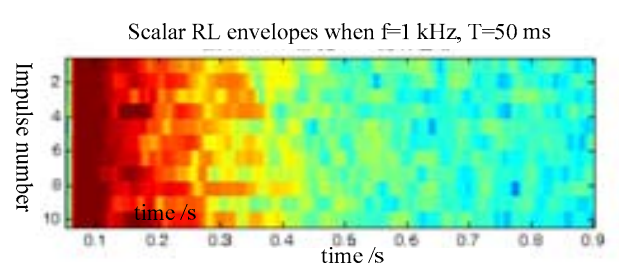

Vector RL envelopes when $\mathrm{f}=1 \mathrm{kHz}, \mathrm{T}=50 \mathrm{~ms}$

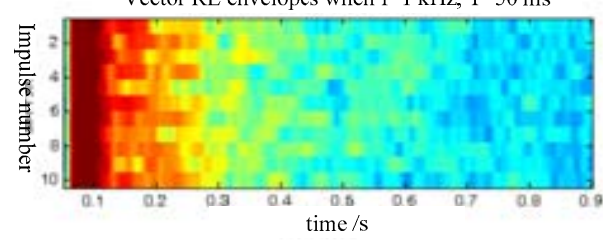

Fig.2.Attenuation of scalar and vector reverberation under signal frequency
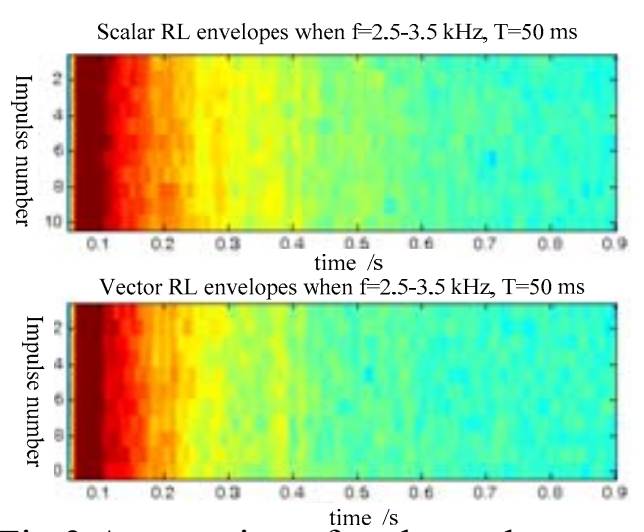

Fig.3.Attenuation of scalar and vector reverberation under broadband frequency

In the monostatic sonar system which is used in the experiment, the head of received signal is direct wave, whose source level is so high that must be useless magnitude-limited when sampling. When calculating reverberation level and researching attenuation laws, the heads must be moved away, Fig 4 shows the reverberation envelopes with direct wave moved whose initial data is the same as Fig 1. 


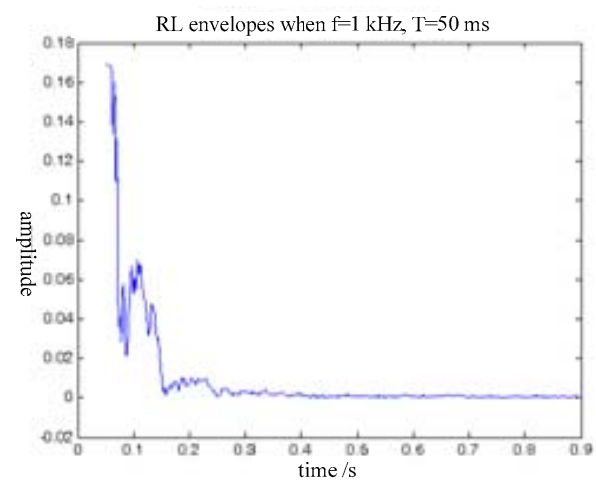

Fig.4.Reverberation envelop with direct wave moved

By comparing RL envelopes with the same transmitting frequency ( $2 \mathrm{kHz}$ ), but different impulse widths(T=20 ms, $50 \mathrm{~ms}, 100 \mathrm{~ms}, 200 \mathrm{~ms}, 500 \mathrm{~ms}$ ), as is shown in Fig 5, It is found that RL envelopes attenuates with time pushing forward, increase with impulse width getting wider. Attenuating laws of vector RL envelopes are similar to those of scalar ones, as is shown in Fig 6, apart from that vector RL envelopes are $5 \mathrm{~dB}$ lower than scalar RL envelopes on average, which due to the nature directivity of vector hydrophones, proving that vector hydrophones have advantages in restraining reverberation.

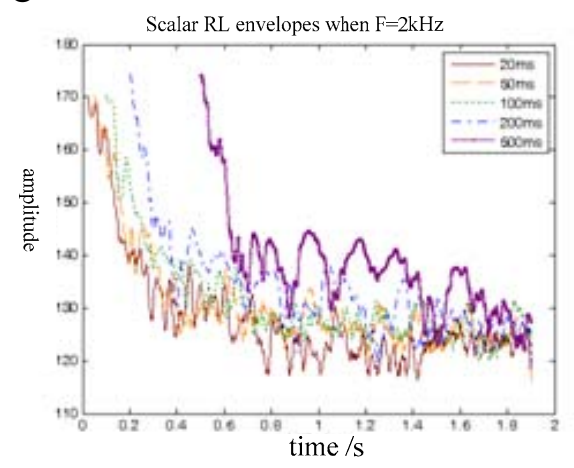

(a) (scalar) pressure RL envelopes

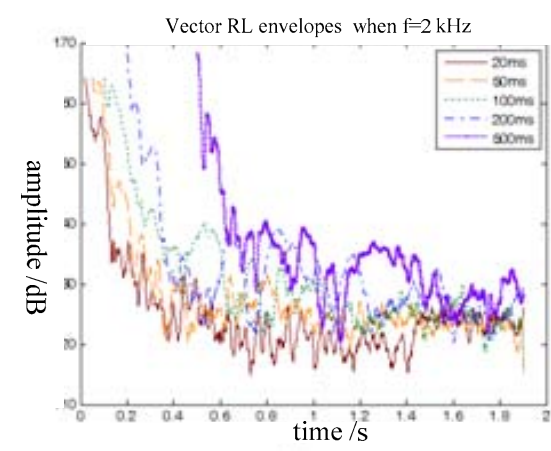

(b) vector RL envelopes

Fig.5. $\mathrm{f}=2 \mathrm{kHz}, \mathrm{RL}$ envelopes

For broadband signals $(2.5 \mathrm{kHz}-3.5 \mathrm{kHz})$ with different impulse widths, similarly processed as simple frequency signals, their RL envelopes show that wider impulse width holds higher RL, and vector RLs are lower than scalar RLs, the only difference to note is that vector RLs are almost 10 $\mathrm{dB}$ lower at the starting time and the discrepancy gets smaller with time and finally vanish when $\mathrm{t}=1.9$ second(as is shown in Fig 6 .

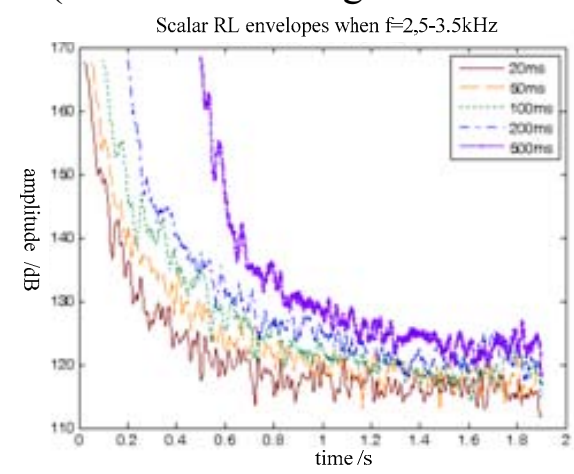

(a) Scalar RL envelopes

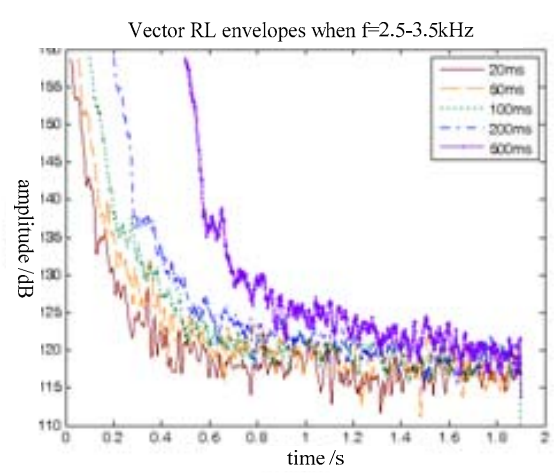

(b) vector RL envelopes

Fig.6. RL envelopes of broadband frequency $2.5-3.5 \mathrm{kHz}$

For broadband signals with impulse width a constant $\mathrm{T}=50 \mathrm{~ms}$, but different frequency scope $(900 \mathrm{~Hz}-1 \mathrm{kHz}, \quad 2.5 \mathrm{kHz}-3.6 \mathrm{kHz}, \quad 2 \mathrm{kHz}-4 \mathrm{kHz}, 3.5 \mathrm{kHz}-4.5 \mathrm{kHz})$, by comparing RL envelopes attenuating laws of scalar ones and vector ones, it is found that the discrepancies among different 
frequency scopes are tiny, apart from that RL of $900 \mathrm{~Hz}-1 \mathrm{kHz}$ is obviously higher than others (as is shown in Fig7).

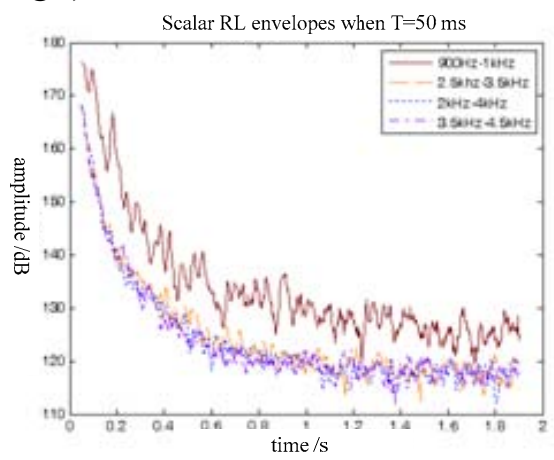

(a) Scalar RL envelopes

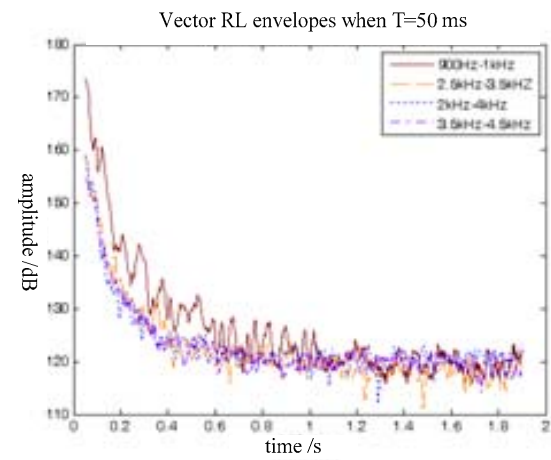

(b) vector RL envelopes

Fig.7. Comparisons of RL envelopes of different frequency scopes

For signals with impulse width a constant $\mathrm{T}=50 \mathrm{~ms}$, but different single frequency $(1 \mathrm{kHz}, 2 \mathrm{kHz}$, $5 \mathrm{kHz}$ ), by comparing attenuation laws of RL envelopes (shown in Fig 8), it is found that for the scalar RL envelopes, the higher frequency, the lower RL , and the faster attenuating RL envelope (shown in Fig 8 (a)), but for the vector RL envelopes it is a little different, in the first 0.4 second of reverberation, the discrepancies among different frequency is quite tiny, since after $\mathrm{t}=0.4 \mathrm{~s}$, RLs of high frequency attenuates faster than that of low frequency, and envelopes of different frequency separate from each other clearly(shown in Fig 8 (b)).

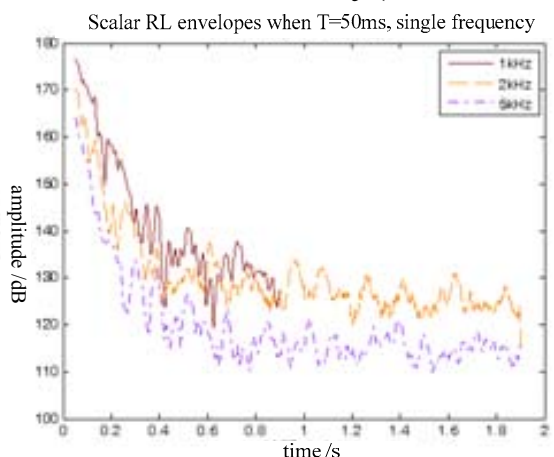

(a) (scalar) pressure RL envelopes

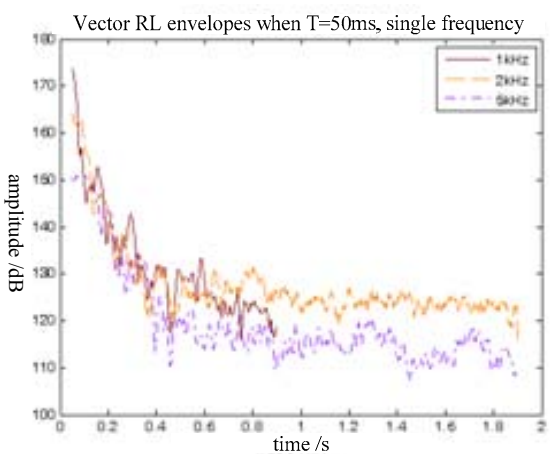

(b) vector RL envelopes

Fig.8.Comparisons of RL envelopes for different frequency signals

In addition, depth of hydrophone affects both the characteristics of reverberation energy attenuation and the performance of underwater devices. In Fig 9, RL envelopes from vector hydrophones at different depths are analyzed. With impulse width $50 \mathrm{~ms}$, transmitting frequency 1 $\mathrm{kHz}$, by comparing attenuating laws of RL envelopes from receiving depths $9 \mathrm{~m}, 9.7 \mathrm{~m}, 10.4 \mathrm{~m}$, $11.1 \mathrm{~m}, 11.45 \mathrm{~m}, 11.7 \mathrm{~m}$, it is found that the discrepancies among RL envelopes from different depths are quite tiny. But this does not mean RL is independent from receiving depth, because the receiving depths vary small, where the reverberation changes have not been shown enough.

\section{Conclusion}

It is showed in the experiment and analyses that the attenuation characteristics of vector reverberation energy are basically the same as those of scalar ones, RL increases with impulse width increasing, RL of higher frequency attenuates faster than lower ones. While an outstanding point is that, due to the nature space directivity of hydrophones, in the same measuring condition, vector $\mathrm{RL}$ is lower than that scalar RL, and energy of vector reverberation attenuates faster than that of scalar ones. Thus in the detection and identification of active sonar system, taking vector hydrophones as receiver has advantages in anti-reverberation. 


\section{References}

[1] Tan Tian, Guo-zhi Liu, Da-jun Sun. Techniques of sonar[M].Harbin. Harbin Engineering University Press,2006.

[2] Jian-jun Xing. Research on the Technique of Direction Detection based on Vector Hydrophone. Northwestern Polytechnical University, Master Degree Dissertation. March 2005.

[3] Gui-qing Sun, Research on Detection and Measurement of Vector Hydrophones, Harbin Engineering University, Doctor Degree Dissertation, May 2001.

[4] Yu Zhao. Research on Array Processing of Vector Array. Harbin Engineering University, Doctor Degree Dissertation, June 2004.

[5] Gui-qing Sun, Qi-hu Li. Acoustic vector sensor signal processing [J].ACTA ACUSTICA, Vol.29,NO.6,2004.491-498. 